\title{
NeUROGRADUALISM: NeURODIVERSITY WITHOUT CATEGORICAL DIFFERENCE, A CASE Study OF AUtism
}

\begin{abstract}
This paper is a study of the link between the phenomenological theme of embodiment and the hermeneutical theme of making (participatory) sense of each other. Using autism as a case in point, I investigate the relation between breakdowns of the latter and neurological diversity. Is trying to explain psychopathologies like autism in this sense not just a way of understanding the human condition as such? In arguing for an emphatic 'Yes!' to this question, I elaborate a case for an empathic neurogradualism. This means that instead of emphasizing the gap between 'neurocultures' we come to an understanding of neurodiversity which informs mutual and selfunderstanding across such cultures. In this view the phenomenology of desynchronization and the cognitive science underlying views on neurodiversity mutually inform each other and neurodiversity becomes a source of inspiration rather than a matter of categorical separation.
\end{abstract}

Keywords

Autism, ASD, neurodiversity, embodiment, enactivism, hermeneutic philosophy, cognitive science, participatory sense-making 
"Orr would be crazy to fly more missions and sane if he didn't, but if he was sane, he had to fly them. If he flew them, he was crazy and didn't have to; but if he didn't want to, he was sane and had to." Joseph Heller, Catch 22 (1961).

\section{Introduction}

Answers to the question 'What is autism?' tend to focus either on the individual bodily (neurological) diversity or on cultural preferences for a specific style of intersubjective coupling leading to marginalization of the autistic population. Both of these research positions assume the validity of a psychiatric diagnosis able to categorize people in a binary way as either autistic or not. The moral implication of such a binary categorization is that it confronts autistic people with a dilemma, reminiscent of J. Heller's satirical novel Catch-22 (1961). Indeed, our ${ }^{1}$ situation under this categorical psychiatric distinction can be paraphrased as a Catch-22: "If we accept to be autistic we are considered crazy, but if we do not accept to be autistic we go crazy." Either we fit in a culturally defined binary category of a diagnosed mental disorder or we show we can adapt to a point where many of us experience a nervous breakdown. The concept of neurodiversity can be seen as attempt to escape from this Catch-22 allowing positive identification with autism as a valuable diverse way of being whilst at the same time recognizing issues that stem from autistic difference.

In Bervoets and Hens (2020), I argued that to go beyond this Catch-22 of autism diagnosis and research, we need to reconceptualize autism. There, I propose to reconceptualize it as a bodily difference, for instance based on the Predictive Coding (PC) theory proposed by Van de Cruys et al. (2014), probabilistically linked to a pathology of intersubjectivity. This approach is in line with what is described by Fuchs (2015) as a difference in embodied intersubjectivity underlying issues of synchronization with other people's perspectives. Understanding autism in this way allows us to make sense of the autistic experience as a vulnerability to desynchronization with respect to conventional social standards of interaction that is far more likely to occur in those who are neurodiverse. It nevertheless is a vulnerability that does not necessarily lead to a pathological breakdown of synchronization, as long as there is a sufficient understanding for this diversity. Indeed, as De Jaegher (2013) shows, difference in embodied intersubjectivity can be overcome via participatory sense-making where autistic and nonautistic individuals do succeed in synchronizing their perspectives. They achieve such a mutual 
understanding despite their differences by attending to (making sense of) their (inter)actions within a shared world, or, in short, by participating in each other's sense-making. My proposed reconceptualization then amounts to simultaneously recognizing a difference in embodiment without equating this difference deterministically to a pathological outcome.

This view leads to some new questions that I intend to address here. On the one hand, in trying to account for the neurodiversity aspect of autism within the new explanatory framework of predictive coding, didn't I risk reducing autism to brain function again? On the other hand, is it possible, and if so how, to connect accounts focused on Erklären (explaining the condition of autism by referring to the bodily or brain dimension) and Verstehen (understanding another's perspective on the intersubjective level).

These questions open a horizon to study, starting from autism as a case in point, the link between the phenomenological theme of embodiment and the hermeneutical theme of making (participatory) sense of each other. The general question then becomes one about the relation between breakdowns of the latter and differences in embodiment, specifically those related to neurological diversity. Is trying to explain psychopathologies like autism in this sense not just a specific way of getting to an understanding of the human condition as such? In arguing for an emphatic 'Yes!' to this question I will elaborate a case for an empathic 'neurogradualism'. An understanding of neurodiversity based on the interplay of neurological differences studied in cognitive science and the phenomenology of desynchronization arrives at a view that stresses mutual understanding, or participatory sense-making, rather than a view of neurodiversity that sets autistics apart as a categorically different 'neuroculture', as is for instance done by Jaarsma \& Welin (2012). Neurogradualism seeks to arrive at a common ground on which diversity can be a source of inspiration instead of a matter of separation. Indeed, breakdowns that are commonly associated with 'autistic brains' then tell us something about the fragile nature of human intersubjectivity. That said, autism research then also might become an example of truly interdisciplinary research, taking into account the dialectic between intra- and intersubjective factors (Bolis et al. 2017).

In section 1, I summarize my proposal to reconceptualize autism, based on a discussion of the mentioned Catch-22. In section 2, I connect this proposal to a general phenomenology of desynchronization as a breakdown of a natural everyday hermeneutical balance of living with 'others' who are irreducibly different from you but all the same fundamentally alike enough to live in an expectation of agreement. With this context, I elaborate in section 3 the notion of neurogradualism, linking the ideas of the human spectrum of neurodiversity and the all too 
human threat of desynchronization. In the last section, I shortly look at the practical implications of this view.

\section{Beyond the Catch-22 of autism research and diagnosis}

There is a tension between the disorder view of autism as codified in DSM-5 (APA 2013) and the positive identity view of it as advocated by the neurodiversity movement (Jaarsma and Welin 2012; Chapman 2019; Bervoets and Hens 2020). In DSM-5, autism is defined behaviorally and, at the same time, coupled to an innate developmental disorder ${ }^{2}$. For a diagnosis, additionally, the criterion of social dysfunction has to be met. From the autistic point of view (specifically in cases, like mine, of being diagnosed with autism as an adult) this means getting entangled in a moral dilemma, in the Catch-22 mentioned above: "If I accept to be autistic I am considered crazy, but if I do not accept to be autistic, I will go crazy." In Bervoets and Hens (2020), I argued that going beyond this Catch-22 requires taking into account the ethical dimension of trying to answer theoretical questions as to 'what autism is'.

This ethical dimension is, in my view, originally present in the very naming of autism. At first glance this naming may be seen as something pejorative, setting people apart. Looking closer however, it may be seen as a natural and useful way to engage with distinct behavioral patterns. In this sense it can be seen as an attempt to understand people who act (or behave) differently, for instance by displaying restricted, repetitive patterns of behavior (see clause B of DSM-5 ASD criteria). Such a fundamentally positive dynamic expresses itself in studying prototypical cases in order to try to make sense of some felt difference. However, this practice subsequently gives way to the drive to capture this difference via diagnostical tests that make a categorical difference strictly segregating between problematic autistic and normal non-autistic behavior ${ }^{3}$. Although the recent terminology of 'spectrum disorder' (APA 2013) gives credit to intracategorial diversity, it does not remove an intercategorial difference between healthy

\footnotetext{
2 The DSM-5 defines Autism Spectrum Disorder (ASD) based on behavioral criteria (i.e. deficits in social communication and repetitive behavior/restricted interests) that need to be present from early childhood and lead to social dysfunction. The category of ASD subsumes as part of the autistic spectrum cases that were previously separately qualified as Asperger's syndrome or Pervasive Developmental Disorder.

Already in the work of Asperger (1944, p.7), where he explicitly resists systematic testing for autism, we see the tension between working from prototypes and defaulting to stereotypes. It is of importance to note here that Asperger saw autism as something common between people "in and out of hospitals".
} 
individuals and autistics categorically diagnosed via dysfunction marked on specific behavioral criteria, see for more detail Bervoets and Hens (2020). The sets of people so defined via behavioral stereotypes are then used to get to the 'bottom' of autism, powering a theoretical drive to explain autistic difference ${ }^{4}$.

This in turn sets the stage for a debate on autism between the exact and human sciences (Hacking 1999). The former try to capture autism in terms of a 'real' difference in neurological structure whilst the latter try to unmask it as a 'social construction' enforcing a contingently preferred intersubjective style. As argued in detail in Bervoets and Hens (2020), both approaches lead to our Catch-22. Indeed, both approaches accept a diagnostic classification that links autism one-to-one to dysfunction. Therefore the choice they leave is one between either being condemned to dysfunctioning because of being "born that way", or, attributing one's dysfunctioning to oppressive processes by the societal majority, leaving affected individuals passive and powerless. The autistic person is then, de facto, put before a choice between being biologically or socially determined, based on opposed but equally general theoretical viewpoints both of which, when taken to the extreme, abstract from the 'lived' autistic experience as an active struggle to cope with a difference, without either accepting its limitations or succumbing to the pressure of denying that difference ${ }^{5}$.

The conclusion is that going beyond the Catch-22 requires a reconceptualization of autism as a bodily difference probabilistically linked to a pathology of intersubjectivity as described by Fuchs (2015). Indeed, this allows positive identification with autism as a valuable diverse way of being whilst at the same time recognizing issues that stem from autistic difference. It is also a way of directly doing justice to the intuitive appeal of the term 'neurodiversity' as a diversity to be understood on the back of a neurological explanation. This is maybe best summarized using a quantum mechanical metaphor. On the one hand, there is a particle aspect to autism in which we try to pinpoint its biological or neurological mechanism via the methods of exact

4 Defining autistics as the set of people with problematic functioning based on a behavioral diagnosis per DSM-5 obviously also begs the question, as it implies that any set of people thus selected to discover an underlying neurological difference eo ipso will exhibit such problematic behavior (Bervoets and Hens 2020).

5 This is not to deny there is a movement both in philosophy as well as in cognitive science to put the autistic experience into the focus of research. Some examples are: Hens and Langenberg (2018) inquiring into the phenomenology of the autistic lived experience, the work of Hanne De Jaegher (2013) on participatory sense-making, the dialectical misattunement hypothesis as is put forward by Bolis et al. (2017) as well as proposals based on predictive coding such as for instance proposed in Van de Cruys et al. (2014). For a more comprehensive review of moral implications of autism research, see Bervoets and Hens (2020). 
science. There is, on the other hand, a wave aspect to autism linked to cultural mechanisms leading up to breakdowns of intersubjectivity, typically studied in the human sciences. The benefit of this metaphor is that it illustrates a difficulty inherent in this reconceptualization. Its indeterminacy is unsatisfying to many, as some believe that, if we just try hard enough we'll be able to pinpoint a bodily cause; whilst others will maintain that no matter how hard we try, it makes no sense to link intersubjective coupling to specific neurological elements ${ }^{6}$.

Nevertheless, mirroring what was said above, it is precisely this indeterminacy that leaves room to autistics to 'own' their autism as a positive identity without accepting that they then, as the widely accepted diagnostic definition has it, need to be considered dysfunctional. It is precisely this indeterminacy that creates room for moral imagination as argued for by Arpaly (2005): it allows to become attentive again to the actual reasons an agent has for behaving in a certain way instead of putting such a behavior down purely to being biologically or socially determined. This creates space for the autistic lived experience, showing that Erklären and Verstehen (explaining autism and understanding autistics) are not at odds with each other but are, instead, intimately related. Any 'explanation' equating behavior with biology destroys this indeterminacy and with it the room for moral imagination that was the initial ethical spur to it in the first place. Instead of understanding the other such explanation will become blind to the autistic lived experience.

For this reason I proposed to rename the DSM-5 category related to autism to Autism Related Disorder (ARD). Here the word 'related' explicity indicates the recognition of autism as a phenomenon independent of pathology whilst also doing justice to the fact many of us need support at some point in our lives. I think it also best embraces the indeterminacy and allows both aspects of autism to be researched in a truly interdisciplinary way (the intra- and intersubjective sides' of autism to borrow the phrase from Bolis et al. (2017)). That said, this proposal also begs the questions mentioned in the introduction. First, it might still be mistaken as implying that the explanation of the bodily aspect of autism is somehow more fundamental than understanding the intersubjective challenges that autistics face ${ }^{7}$. Second, it does not show

6 In Bervoets and Hens (2020), we arrive at the indetermiancy by appeal to a middle position in the (anti-)physicalism as proposed by Davidson (2001a). This middle position, called Anomalous Monism, maintains that all mental events are related to physical events but can never be brought back to them in a nomological or law-like way that connects mental events in a one-to-one fashion to physical events.

7 I thank an anonymous reviewer for pointing out that 'Autism Related Disorder does not imply a biomedical attitude but only that there is a link (albeit a non-deterministic one) between autism and a 
how to bridge between accounts focused on Erklären (explaining the bodily or brain dimension) and Verstehen (understanding the other intersubjectively). This creates the risk to, again, oppose ideas of autism as a neutral neurological variation from ideas of social construction of problematic autistic behavior. To avoid falling into such a dichotomy again it is not sufficient to critically analyze autism research but necessary to create a constructive account of how the bodily and the intersubjective (behavioral challenges) element interact, a Schrödinger equation of neurodiversity if you will.

\section{Linking desynchronization and difference in embodiment as a step towards mutual understanding}

In tackling such a constructive account, it seems more natural to think about it in terms of the phenomenological (enactivist) theme of the body and the hermeneutical theme of interpreting (making sense of) the other ${ }^{8}$. Roughly, I set out to respectively link the intra-subjective and the intersubjective aspects of autism to the enactivist tradition and to the hermeneutical philosophy of Gadamer. The former suggests itself naturally as a way to do justice to an understanding of the lived experience of autism, certainly insofar it is related to a difference in embodiment. The latter seems promising because it is an account of how we can come to a mutual understanding despite differences in our respective 'horizons'. Horizons in Gadamer's sense are a totality of outlook of a specific person given the specific historicity or experiential background of that person, including any preconceptions or prejudices one may have come to have. In the present case the differences in 'horizon' are due to different experiential outlooks tied to differences in embodiment related to autism as is detailed below. The enactive account then shows how a difference in embodiment leads to breakdowns of intersubjectivity that are in turn central to understanding psychopathologies like autism (Fuchs 2015). A hermeneutical account shows that breakdown of intersubjectivity is not specific to psychopathology but a basic element in how we mutually interpret or understand each other despite different life histories. Achieving intersubjectivity or synchronization - the hermeneutical concept of 'fusion of horizons' - may

need for support. That said, the reality is that the biomedical implicature is the most readily made at the present time.

8 In the light of the focus on mutual understanding, that is seen as being served by explanation, two major traditions in continental philosophy elaborating the notion of Verstehen are chosen as suitable candidates for a constructive account. The critical account in the previous section on the other hand based itself naturally on resources from analytic philosophy focused on (the limits of) the notion of Erklären. 
then be particularly challenging in autism but it is not different in kind to the basic hermeneutical situation universally shared by all human beings that aim at understanding one another. It is therefore not necessarily so that autism leads to a lasting breakdown of intersubjectivity. Indeed, de Jaegher (2013) shows how participatory sense-making allows, even in extreme cases of autistic peculiarity of expression, to establish or restore intersubjectivity. However, this implies effort on both sides of the dialogue, in line with the mentioned hermeneutical concept of a 'fusion of horizons'. It is then also not possible to associate a breakdown of intersubjectivity exclusively on the side of the autistic individual. Responsibility for (de)synchronization is shared by the interlocutors ${ }^{9}$, and it cannot be reduced to the atypical embodiement of one party. Hermeneutics in this way allows to express in a more universal way Milton’s ‘double empathy problem' (Milton 2017) which is so central to recent accounts of neurodiversity.

I believe introducing hermeneutics helps to clarify matters, as it highlights intersubjectivity as a given background assumption, as the natural everyday balance of living with 'others'. It highlights our everyday expectation of - or trust in - finding agreement despite our differences (whatever sort of a difference that may be). When that expectation is not met, we experience a moment of desynchronization where our differences become salient. We have to put effort where effortless balance is the rule. I propose this moment of awkwardness is inherent in desynchronization, and is a moment which either can be taken as an incitement to exercise one's moral imagination leading to a 'fusion of horizons' or can be the starting point of a pathological breakdown of intersubjectivity. A sustained period of such moments of desynchronization cannot but lead to a violation of trust which, following Ratcliffe (2017), is itself a common characteristic of mental illness ${ }^{10}$. In autism specifically this violation of trust, as a violation of predictability in the environment (see below), leads to the coping mechanism of increasing the predictability of or control over one's environment in developing repetitive and restricted behaviors thematized in the DSM-5 (APA 2013; Constant et al. 2018). Carving out such idiosyncratic patterns may itself constitute a historicity that complicates any further 'fusing of horizons'. That said, every moment of desynchronization also provides the impetus

9 This is not to be interpreted in a strict way but includes the societal responsibility for creating conditions where specific people can bridge the gap. Crucially this includes explanations about why a specific embodiment creates specific challenges. An elaborate account of responsibility is beyond the scope of this paper but neurogradualism is, on this view, a spur to be more imaginative in such accounts. 10 Where Ratcliffe discusses this in the specific case of psychotic phenomena the applicability to autism can be seen for example via the side-by-side treatment of problems of intersubjectivity in autism and schizophrenia in Fuchs (2015). 
for moral imagination in trying to make sense of, or understand, an awkward situation. Doing so however requires understanding why such moments occur in the specific case of autism to which we turn next.

One reason for such moments may well prove to be that the other's body does not react to the world in the same way your body does. If such is the case, this will be a cause for a persistent occurrence of such moments. Although there are rather immediate ways to see whether one's bodies are different, this is not always the case. Even in the cases of blindness or deafness it would seem that realizing such a difference passes through that kind of a moment of desynchronization ${ }^{11}$. Indeed, both temporary and permanent deafness are first detected as the inability to communicate via default acoustic means of synchronization. It is only in second order that we try to establish other ways of communication given the mutual recognition that it is deafness that stands in the way of mutual understanding in the default way. Re-establishing communication crucially depends on explaining the respective horizons of the interlocutors attempting to understand each other. Indeed, the differences between temporary and permanent deafness consist in explaining how it came about as well as the expectation of what is to come. In case of permanent deafness there likely is the alternative of sign language or lip reading whilst in temporary deafness such alternatives are unlikely, nor are they likely to be worthwhile to develop. This history and expectation is, by the way, not just important on the side of the deaf person, because if more people were versed in sign language this alternative might well be a more generally available element of fusing horizons. Here we see the intimate relationship between Verstehen and Erklären again: the way we reestablish understanding necessarily passes via the analysis (the explanation) of why the moment of desynchronization happened at all (and such explanations are never fully one-sided with respect to the capabilities of one person!).

In short, moments of desynchronization spur the imagination in order to reestablish a mutual understanding. This imagination is first and foremost moral imagination (Arpaly 2005), which connects to what was said above on the ethical dimension being originally present in the very naming of autism, as an attempt to understand people who behave differently. In this regard autism may be an exemplary, prototypical, case of such sustained experience of desynchronization. Autism then defies the imagination, exactly because no visible bodily or

11 Once people living with you get used to the impacts of the difference, such moments will tend to be less frequent and less intrusive and this points to a way of healing/recovery through 'familiarization' which, for lack of space, I cannot develop here. 
cultural difference can be found even if it persists throughout an autistic lifetime with a characteristic style of being. ${ }^{12}$ This would account for the lasting spur to imagination autism has, for it is both something strange that confronts us from within our cultures and appearance and, therefore, something that, because it complicates mutual understanding, incites scientific curiosity for a helpful explanation. In this light it is really important (as it was already important in Asperger's (1944) account) that a link to some hereditary, neurological or innate, difference has been implied for autism.

This, I think, is at least a sketch of how to bridge accounts focused on (bodily) explanation and (intersubjective) understanding. The wave aspect - persistence of desynchronization - and the particle aspect - the assumption of neurological diversity - cannot be fully disambiguated. The dynamic is as follows: persistent desynchronization leads to efforts of hermeneutical Verstehen in the sense of trying to (re)establish synchronization; part of this effort lies in explaining - in Erklären - a cause of the intersubjective breakdown (for instance in differences in embodiment or neurology) and this in turn leads to enactive Verstehen - in the sense of participatory sensemaking - of how neurological differences impact each other's lived experience. If the end result is successful - there is a mutual understanding or fusing of horizons - then both parties of the intersubjective exchange have effectively adapted (their horizon), meaning that Verstehen and Erklären as one indivisable hermeneutical dynamic can never be fully separated from one another.

This means that we are back to the indeterminacy with which I ended section 2, although we now see that the basis for the quantum mechanical metaphor is a hermeneutical gap. Indeed, the understanding of autism in terms of the indeterminacy is based in the need to always put in active effort to see the other's perspective (fusing horizons is never a given, never a finished process). The naming of autism is, then, nothing but the attempt to understand people who have a vulnerability for recurring moments of desynchronization from early childhood whilst not being visibly different. As we make sense of autistics (and therefore also explain autism to a certain extent), it is possible to fuse horizons between autistics and non-autistics, thereby preventing the persistence of such moments and their development into - what is deemed to be - problematic behavior. All this means that from a methodological point of view, autism can only be understood by an interdisciplinary cooperation between the human and exact sciences

12 See for instance the use of the word "Ausdruckserscheiningen" by Asperger (1944, p. 7). He employs the term "Ausdruckserscheinungen" in opposing the approach of creating stereotypical "test machinery" that aims to explain autism on the basis of an a priori theoretic system (see also note 3 ). 
as it has the characteristics, in the terms of Hacking (1999) of both a human and a natural kind. Autism cannot be fully determined by either its neurology or its social construction but requires both elements, for more detail on the indeterminacy of autism see Bervoets and Hens (2020)). It is important to note here how the discomfort with indeterminacy leads to two reactions that destroy in different ways the intricate relationship between Verstehen and Erklären. One is to explain it away by making the difference visible, e.g. by locating autism in a person's genetic makeup or by defining autistics according to behavioral characteristics which are deemed to be dysfunctional. This reaction leads to positing a categorical diagnostic distinction between two types of embodiment or behavior without recognizing the possibility of exercising both sides' moral imagination to actively bridge the hermeneutical gap. It closes horizons ${ }^{13}$ and it does so in a way pathologizing those who have atypical intersubjective preferences. The other reaction is to extend the methodological point that the concept autism is a moving target to calling into question the bodily reality of it. In doing so, understanding autism is reduced to understanding problematic behavior and is cut off from any explanation as to how such moments of desynchronization come about in the first place. Without an explanation based on (bodily) differences, intersubjective breakdowns occurring for that reason cannot be prevented. This turns into an ethical deficit, as well as stripping people of cultural and scientific bridges that help them fuse horizons. It stands in the way of fusing horizons, since it prevents seeing commonality of lived experience (or phenomenology) across situations. It does so by making autism specific to a particular cultural and historical situation, thus preventing autistics to identify cross-culturally and cross-historically.

\section{Neurogradualism: diversity without categorical difference}

This finally brings me to the title of this paper: neurogradualism. One may indeed wonder if all this indeterminacy does not bring us close to one of the reactions most hated by autistics: "Isn't everybody then a little autistic?" This question seems to deny one key tenet of the neurodiversity movement related to the 'neuro'-part of the term - see, for instance, Jaarsma and Welin (2012) - stressing autism as a categorical biological difference out of the control of the neurodiverse individual. From this point of view autism is sometimes seen as 'neuroculture'

\footnotetext{
13 It is interesting to note the parallel between the unsustainability of closed horizons as per Gadamer and an impossibility, as noted by Davidson (2001b), to make sense of the notion of (separate) conceptual scheme(s). It seems that this analytic and continental approach to language lead to a similar conclusion.
} 
or 'neurotribe', a closed-off community. The other key element in neurodiversity relates to the 'diversity'-part of the term. This clearly refers to another key tenet of the movement: a striving for understanding, resulting in inclusion and acceptance as a positive, albeit minoritarian, identity. Our solution resolves the tension between the tenets by denying the categorical aspect of the biological difference. Still, in the spirit of indeterminacy, it is not so that biological difference is belittled. Far from it, it has, as is clear from what I argued for above, a central place in this account. The contribution of this proposal then is that it integrates the 'neuro'-part of neurodiversity without basing a categorical distinction on it. This grounds on the fact that such neurological difference can be overcome in our intersubjective practice by fusing horizons via participatory sense-making. It allows us to embrace autism as a positive identity, whose identity-holders have the rightful claim to be accepted "as they are", without being categorically set apart. It so principally allows that biological difference can be overcome by individual and collective action and thus enables that the ethical primacy of mutual understanding trumps that of setting autistics categorically apart. This is achieved without denying that autistic embodiment gives rise to specific intersubjective challenges that may require special support given the situations and environments autistic individuals find themselves in.

The more charitable reading of "Isn't everybody a little autistic?" then is that in trying to explain psychopathologies like autism, we are just trying to understand the human condition as such. Autism, as I tried to capture it in the section before, is an extreme version of an all too human hermeneutical situation that, on a background of trust, we always also are dependent on others and, therefore, vulnerable to moments of desynchronization. Still, there is a difference between having an autistic moment and being autistic (maybe much in the same way as there is a difference between being deafened and being deaf). Autism, that much is clear from the neurodiversity movement, is felt as a persistent problem of tuning to the horizon of others. It is not something that is felt to be dependent on circumstances, but it is a different 'way of being' or 'form of life', anchored in a different embodiment. It is, however, something that only becomes pathological when an individual tuning gap cannot be bridged by participatory sense-making. This does not mean that every gap on account of an autistic biological difference will be detected (therefore also a great many neurodiverse individuals will not be diagnosed or only diagnosed later in life). The latter will depend on both the resources for detection - which may be few and far between in developing countries or certain sets of the population or simply biased against e.g. detecting autism in women - and on individual support needs - based for instance on one's immediate personal environment and other biological or socioeconomic 
challenges. The parallel with deafness can, I think, be upheld here as well as there was and is no guarantee that it will always be detected as the root cause of problems of intersubjectivity; although it is surely so that when it is detected it can be a crucial element of better inclusion. Whether or not an autistic difference then leads to (detection of) dysfunctioning in the psychiatric sense $i$ s something that does depends on a specific (individual) history and cultural environment. When we, for instance, societally enforce too narrow a 'neurotypical' horizon in the workplace or in school, we will exclude a larger group. This will result in having to roll out more special support or, as is unfortunately still more often the case, marginalizing more people, specifically those who are vulnerable, because of, for instance, socio-economic reasons. Another example may be that of an autisic individual who is fortunate enough to have a more conducive environment or more resources to compensate for their difference. In all this we find the indeterminacy again. Indeed, autistic difference is not sufficient in itself to meet (specifically) the dysfunctionality criterion of DSM-5 ASD even if it is probabilistically linked to the other criteria (see my proposal in section 1 to rename ASD to ARD, Autism Related Disorder).

The idea of neurogradualism then is to not neatly cut off autistics from non-autistics and at the same time still study what kind of difference in enbrainment can lead to such a persistent tuning problem. I proposed (Bervoets and Hens 2020) a way of validating a predictive processing account of the particle aspect of autism (an account as was proposed in Van de Cruys et al. (2014)), focusing on the parameter of precision. On this proposal human perception is generically characterized by comparing 'bottom-up' sense-data with prior 'top-down' expectations. The comparison then yields Bayesian evidence for one or another of these expectations. How one weighs the prediction error is reflected in the precision parameter with which one makes this comparison. This parameter is crucial in this approach as it directly relates to how precisely this comparison needs to 'fit' in order for the process to come to closure. Autistic difference on the account of Van de Cruys et al. (2014) is related to a higher and more inflexible setting of the precision parameter.

My solution of neurogradualism can then be read as this parameter being gradually different in human beings. If set in a too 'High and Inflexible' way (given a certain environment) this can lead to an intersubjective breakdown or continuous alertness to moments of desynchronization (leading e.g. to burn-out). Such an account cannot lead to a categorical 'autism' cut-off. Even if there are specific behavioral patterns associated with it (as for instance argued in Constant et al. (2018)), their development into or appreciation as dysfunctionality will not only depend on this individual precision parameter. Indeed, if somebody with a precision that is more 'High 
and Inflexible' encounters environments that are less forgiving of the differences in behavioral tendency that go with it, they will be more rapidly perceived as dysfunctional. However, if they would find themselves in a more inclusive environment, they would be much less likely to be considered dysfunctional or encounter personal issues with their 'way of being'. This means, in line with the 'ARD' proposal, that dysfunctionality (rather than being autistic!) is dependent on situation or context and specifically on how 'ableist' ${ }^{14}$ one's environment is in the sense of enforcing 'neurotypical' behaviors like looking one's interlocutor in the eye, not 'stimming' and so on.

The above also leaves open that precision weighting is a common developmental pathway (Johnson 2017) associated to multiple genetic causes and/or a dynamic parameter influenced by environmental factors. As to the latter, the specific source of dynamism may lie in epigenetic mechanisms known to tie environmental factors to impact a person's biology, for example via stress (Denhardt 2017). In this way we can see how a propensity for atypical precision may be either alleviated or aggravated by the stress one encounters in one's life history. Indeed, if one can cope with circumstances this atypicality may remain relatively neutral, whereas once one has experienced a significant break-down in trust - such as in trauma - this may lead to a period of hyperattentiveness in which atypical precision is exacerbated to a point of unavoidability of dysfunction. This underlines the entanglement of individual differences and the way a society or one's surroundings are crucial in dealing with an individual's atypicality in such a way as to avoid things to spiral out of control. Although I cannot work this out in scope of the present paper, these types of connections are a strength of our reconceptualization, as they allow to empirically investigate questions about autism as a common developmental pathway and/or the epigenetic links between autism and e.g. post-traumatic stress disorder.

Neurogradualism then allows to connect the general phenomenology of desynchronization to the cognitive science related to neurological diversity without reducing the one to the other. It connects the behavioral and the biological without reducing the breakdowns of intersubjective coupling to one side of that intersubjective equation and reducing that party to the biology or neurophysiology claimed to be inherently pathological. Neurogradualism capitalizes on the insight of the enactivist tradition to see a moment of desynchronization as always also a sign of an underlying difference in embodiment. It, however, does so avoiding the risk of stating,

14 The notion of ableism is generally used to indicate the extent to which a society excludes people who are not able to behave in ways that people majoritarily behave. Originally used in the context of physical disability, its use now is accepted in the case of mental 'disability'. 
as for instance done by Hutto (2003), that such a difference can in principle be insurmountable, splitting people into clean cut and herme(neu)tically sealed off kinds.

\section{Conclusion}

In "The Enigma of Health" 15 Gadamer (1993) characterizes the doctor as asking a prototypical question: "What do you feel is wrong with you?". The autistic may well live most of her life answering: "Nothing, really." - as long as her moments of desynchronization do not lead to the belief that she cannot get back to the safe (charitable) place of understanding others and being understood by them. Indeed, mirroring the words of Davidson (2001b), what more needs to be expected of intersubjective coupling than successfully referring to a shared world applying the Principle of Charity in doing so? If anything, the present paper asks not to prejudge another as dysfunctional but to maximally strive for participatory sense-making as proposed by de Jaegher (2013). Hermeneutical success may not be guaranteed but it surely always is the human(e) thing to aim for.

One may well complain that neurogradualism is too messy a solution to the problems set forth by neurodiversity, as it is well possible that there are people with a certain bodily difference (e.g. related to precision weighting) who will never be diagnosed. In fact, my proposal to rename ASD to Autism Related Disorder whilst keeping the criteria intact explicitly leaves open a possibility that someone with a same bodily difference as individuals who are diagnosed (following perceived dysfunction) does not experience dysfunction, as they find themselves in a more inclusive environment. In parallel, people with similar behavioral tendencies may or may not experience dysfunction (feel like something is wrong with them given their intersubjective context) and their behavior will not be deterministically linked to a specific bodily parameter like precision weighting. To expect to fully close this explanatory gap would be - based on my argument above - not only impractical but also unethical. We can at most expect that there is a probabilistic link between the bodily difference (the autistic nature, the particle aspect of autism) and certain behavioral patterns (the autistic behavior, the wave aspect of autism) that can lead to intersubjective breakdown and the assessment of dysfunction. Keeping these aspects clearly separate is important to keep the proper focus on why persistent desynchronization happens so as to be able to better prevent it, and to avoid wrong explanations

15 This is the official translation of "Über die Verborgenheit der Gesundheit" which, I believe, does not bring out adequately the gist of this collection of essays. I would prefer "On the hiddenness of health", which is anyway close to the interpretation I am making of it. 
(like neurological deficit or behavioral abnormality) that get in the way of inclusion of people who 'feel' neurodiverse. Still, with Gadamer, we should be wary to decide on behalf of others whether or not their 'way of being' is pathological - whether or not they (should) feel something is wrong with them - as, so we argued, desynchronization is a fact about 'us' rather than only about 'them'. The only goal of naming somebody (or yourself) autistic is to make progress in mutual understanding. What I have proposed leaves open the critical space of identifying as autistic without committing oneself to being dysfunctional in a certain way. In the spirit of Gadamer, we can say that correct scientific explanations are only a part of that, albeit an important part, as is clear from the above and the expanding interest in interdisciplinary research on autism.

In conclusion, maybe autism as driving the inclusion of an intra-cultural difference can become an example for research in overcoming intercultural differences. Attributing problems of intersubjectivity to some 'categorical' difference is just an excuse not to do the effort of fusing horizons. Truly respecting diversity, however difficult, always calls for a mutual effort to fuse horizons.

Acknowledgements:

The above would have been impossible without the questions raised by Katrien Schaubroeck, Walter Van Herck and Kristien Hens in first presenting the ideas summarized in section 1. I also thank Rob Sips, Emma Moorman, and, again, Kristien Hens for intensively reviewing previous versions of the paper allowing me to vastly improve it. I also thank the audience at the DGAP workshop held on 11/09/18 in Heidelberg, specifically Hanne de Jaegher for, their insightful questions. Finally, I thank the reviewers of this paper; I do not know whether this is a good paper but I do know it is a much better paper thanks to their comments.

This work was partly funded by the NeuroEpiGenEthics project that received funding from the European Research Council (ERC) under the European Union's Horizon 2020 research and innovation programme (grant agreement No 804881).

References:

American Psychiatric Association. 2013. Diagnostic and Statistical Manual of Mental Disorders (DSM-5®). American Psychiatric Pub.

Asperger, Hans. 1944. “Die „Autistischen Psychopathen” Im Kindesalter.” Archiv Für Psychiatrie Und Nervenkrankheiten 117 (1): 76-136.

Arpaly, Nomy. 2005. "How It Is Not 'Just Like Diabetes': Mental Disorders and the Moral Psychologist1.” Philosophical Issues. A Supplement to Nous 15 (1): 282-98. 
Bervoets, Jo and Hens, Kristien. 2020. "Going Beyond the Catch-22 of Autism Diagnosis and Research. The Moral Implications of (Not) Asking "What Is Autism?"." Front. Psychol. 11:529193. doi: 10.3389/fpsyg.2020.529193

Bolis, Dimitris, Joshua Balsters, Nicole Wenderoth, Cristina Becchio, and Leonhard Schilbach. 2017. "Beyond Autism: Introducing the Dialectical Misattunement Hypothesis and a Bayesian Account of Intersubjectivity." Psychopathology 50 (6): 355-72.

Chapman, Robert. 2019. "Autism as a form of life: wittgenstein and the psychological coherence of autism.” Metaphilosophy 50, 421-440.

Constant, Axel, Jo Bervoets, Kristien Hens, and Sander Van de Cruys. 2018. "Precise Worlds for Certain Minds: An Ecological Perspective on the Relational Self in Autism." Topoi. An International Review of Philosophy. https://doi.org/10.1007/s11245-018-9546-4.

Czech, Herwig. 2018. "Hans Asperger, National Socialism, and 'race Hygiene' in Nazi-Era Vienna." Molecular Autism 9 (1). https://doi.org/10.1186/s13229-018-0208-6.

Davidson, Donald. 2001a. "Mental Events.” In Essays on Actions and Events, 207-28. 2001b. "On the Very Idea of a Conceptual Scheme.” In Inquiries into Truth and Interpretation, 183-98.

Denhardt, David T. 2018. "Effect of stress on human biology: Epigenetics, adaptation, inheritance, and social significance.” J Cell Physiol. 2018 Mar;233(3):1975-1984.

De Jaegher, Hanne. 2013. "Embodiment and Sense-Making in Autism." Frontiers in Integrative Neuroscience 7 (March): 15.

Fuchs, Thomas. 2015. "Pathologies of Intersubjectivity in Autism and Schizophrenia." Consciousness Studies 22 (1-2): 191-214.

Gadamer, Hans-Georg. 1993. Über die Verborgenheit der Gesundheit: Aufsätze und Vorträge.

Hacking, Ian. 1999. The Social Construction of What? Harvard University Press.

Heller, Joseph. 1961. Catch-22. New York: Simon and Schuster.

Hens, Kristien, and Raymond Langenberg. 2018. Experiences of Adults Following an Autism Diagnosis. Palgrave Macmillan.

Hutto, Daniel D. 2003. "Folk Psychological Narratives and the Case of Autism." Philosophical Papers 32 (3): 345-61. 
Jaarsma, Pier, and Stellan Welin. 2012. "Autism as a Natural Human Variation: Reflections on the Claims of the Neurodiversity Movement." Health Care Analysis: HCA: Journal of Health Philosophy and Policy 20 (1): 20-30.

Johnson, Mark H. 2017. “Autism as an Adaptive Common Variant Pathway for Human Brain Development.” Developmental Cognitive Neuroscience 25 (June): 5-11.

Milton, Damian. 2017. “A Mismatch of Salience: Explorations of the Nature of Autism from Theory to Practice." West Sussex, Pavillion.

Ratcliffe, Matthew. 2017. Real Hallucinations: Psychiatric Illness, Intentionality, and the Interpersonal World. MIT Press.

Van de Cruys, Sander, Kris Evers, Ruth Van der Hallen, Lien Van Eylen, Bart Boets, Lee deWit, and Johan Wagemans. 2014. "Precise Minds in Uncertain Worlds: Predictive Coding in Autism." Psychological Review 121 (4): 649-75. 